\title{
Factors Associated With Falls in Older Patients With Diffuse Polyneuropathy
}

\author{
James K. Richardson, MD
}

OBJECTIVES: To identify clinical factors associated with falls by older persons with polyneuropathy (PN).

DESIGN: A cross-sectional study of 82 subjects aged 50 to 85 with clinical and electrodiagnostic evidence of PN.

SETTING: Electrodiagnostic and biomechanical research laboratories.

PARTICIPANTS: Patients referred to the electrodiagnostic laboratory.

MEASUREMENTS: History and physical examination, including semiquantitative methods of peripheral nerve function, and clinical balance testing. Falls were defined by retrospective self-report over a 2 -year period.

RESULTS: Forty (48.8\%), 28 (34.1\%), and 18 (22.0\%) subjects reported a history of at least one fall, multiple falls, and injurious falls, respectively. Factors associated with single and multiple falls were similar, so only results for multiple and injurious falls are reported. Bivariate analysis showed that an increased body mass index (BMI) and more severe PN (as determined by the Michigan Diabetes Neuropathy Score) were associated with both fall categories. Men reporting falls also demonstrated a decreased unipedal stance time. Age, sex, nerve conduction study parameters, Romberg testing, medications, and comorbidities were not consistently associated with either fall category. Logistic regression demonstrated that multiple and injurious falls were associated with an increased BMI and more severe PN, controlling for age, sex, medications, and comorbidities (pseudo $R^{2}=0.458$ and 0.484 , respectively).

CONCLUSIONS: Although previous work has demonstrated that all older persons with PN are at increased risk for falls, patients with increased BMI and more severe PN

From the University of Michigan Medical Center, Department of Physical Medicine and Rehabilitation, Ann Arbor, Michigan.

Supported by Public Health Service Grants K23 AG 00989-01 and 1P30 AG 08808 .

Address correspondence to James K. Richardson, MD, Associate Professor, University of Michigan Medical Center, Department of Physical Medicine and Rehabilitation, MPB D5200, Ann Arbor, MI 48109. E-mail:

jkrich@umich.edu are at particularly high risk and should be targeted for intervention. J Am Geriatr Soc 50:1767-1773, 2002.

Key words: balance; body mass index; falls; geriatric; polyneuropathy

A lthough only recently recognized, the relationship between a diffuse polyneuropathy (PN) and falls is likely neither a new nor a modern phenomenon. Rate of fracture in a medieval British leprosy hospital skeletal sample was found to be about four times that in a control medieval skeletal sample. This finding led the authors to conclude that persons with leprosy, a common cause of $\mathrm{PN}$ in the medieval population studied, sustained more fractures than the control population due to "accidental falls." ${ }^{1}$ Studies of living subjects have demonstrated that patients with PN, which reduces sensation and often strength in the lower extremities in a distal to proximal fashion, have impairments in balance $e^{2-5}$ and an increased risk for falls ${ }^{6}$ and falls causing injury. ${ }^{7,8}$

Furthermore, it appears that the prevalence of diabetes mellitus, and therefore PN, in older persons is increasing. Epidemiological studies suggest that diabetes mellitus and impaired glucose tolerance affect more than $40 \%$ of U.S. citizens aged 60 to $74 .{ }^{9}$ Other work suggests that the prevalence of $\mathrm{PN}$ in this age group is between $32 \%$ and $50 \%$ for persons with diabetes mellitus, $11 \%$ for persons with impaired glucose tolerance, and $7.1 \%$ for normoglycemic persons. ${ }^{10}$ Taken together, these data suggest that the prevalence of PN in those aged 60 to 74 is approximately $22 \%$ and likely to increase.

Given the magnitude of the clinical problem of falls among older persons and the increasing prevalence of PN, an improved understanding of which patients with PN are at greatest risk for falls would be welcome. Although it is clear that older persons with $\mathrm{PN}$ are at increased risk for falls, not all older persons with PN fall. The purpose of this study was to analyze a group of older adults, all with PN, and compare clinical features associated with subjects who reported falls with features of those who did not. The initial hypotheses were that increased age, increased severity of PN, and decreased unipedal stance time (UPS) would be associated with any fall, multiple falls, and injurious falls. 


\section{METHODS}

\section{Subjects}

The PN subjects were examined as part of a screening process for other studies that investigated the effects of PN on postural stability in older persons. Their primary physicians had referred most of the PN subjects to the electrodiagnostic laboratory for symptoms suggestive of a lower extremity peripheral neurologic disorder. A smaller number of the PN subjects responded to local advertisements requesting subjects with neuropathic symptoms and then underwent electrodiagnostic testing. All subjects underwent electrodiagnostic testing in the same laboratory, using the same techniques, by technicians certified by the American Association of Electrodiagnostic Technologists with the supervision of a physician board-certified by the American Association of Electrodiagnostic Medicine.

Inclusion criteria were age between 50 and 85 , symptoms consistent with PN, ability to speak and understand English, ability to ambulate household distances without an assistive device, corrected vision not worse than 20/50, physical examination consistent with PN (absent or decreased Achilles reflexes, decreased distal lower extremity sensation (vibration, pinprick, and light touch) that improved proximally), and electrodiagnostic evidence of a diffuse, primarily axonal $\mathrm{PN}$ as evidenced by:

Sural responses-absent or decreased amplitude $(<6 \mu \mathrm{V})$ with a normal or minimally prolonged distal latency $(<5.0 \mathrm{~ms})$ stimulating $14 \mathrm{~cm}$ proximally from the recording site posterior to the lateral malleolus.

Peroneal and/or tibial motor responses-absent or decreased in amplitude $(<2 \mathrm{mV}$ for peroneal and $<3$ $\mathrm{mV}$ for tibial) with a normal distal latency $(<6.2 \mathrm{~ms}$ stimulating 9 and $8 \mathrm{~cm}$ proximally from recording sites over the extensor digitorum brevis and abductor hallicus muscles, respectively).

If bilateral sural responses were absent, motor responses were not always performed. If the sural responses were present but reduced in amplitude, motor responses were required and must also have been of reduced amplitude. Needle examination was normal or demonstrated findings consistent with PN.

Exclusion criteria were evidence on physical examination of central neurological dysfunction (e.g., hemiparesis, cerebellar dysfunction, Parkinson's disease); musculoskeletal abnormality, such as severe scoliosis or amputation; or electrodiagnostic evidence of a lower extremity abnormality other than PN, such as a myopathic process or radiculopathy.

\section{History and Physical Examination}

Each subject's age, height, weight, sex, and medications were recorded. Past medical history, with particular attention paid to neuromuscular and musculoskeletal conditions that might influence falls, ${ }^{11}$ was recorded as well. Medications considered to be associated with falls included antihypertensives, phenothiazines, benzodiazepines, tricyclic antidepressants, and antiarrhythmics. ${ }^{12}$ A history of falls over the previous 2 years was obtained, with a fall being defined as an unintentional change in body posture that resulted in the subject coming to rest on the ground or other lower level that was not a consequence of a physical blow or loss of consciousness. Falls associated with presyncopal or syncopal symptoms were not included. In the majority of the subjects, a spouse or family member was available to confirm the history. An injurious fall was defined as a fall that caused the subject to seek and receive medical treatment. If the subject sought medical evaluation, but no significant treatment beyond an analgesic for bruising was given, the fall was not considered injurious.

All of the subjects $(\mathrm{N}=82)$ underwent a physical examination that included muscle stretch reflexes, plantar responses, finger-to-nose and heel-to-shin maneuvers, rapid alternating movements of the hands and feet, pronator drift, assessment of rigidity or spasticity by passive range of motion, Romberg testing, evaluation of ankle dorsiflexion strength, and semiquantified vibratory and position sense testing. In addition, the majority of the subjects underwent unipedal stance testing, clinical evaluation of ankle dorsiflexion strength $(\mathrm{n}=71)$ and evaluation with the Michigan Diabetes Neuropathy Score (MDNS; $\mathrm{n}=62$ ). The specifics of the examinations include:

1. Patellar and Achilles muscle stretch reflexes. Efforts to obtain the Achilles reflex included striking the tendon itself and the plantar strike technique. The latter has been found to be more reliable in older persons. ${ }^{13}$ The reflex was considered present if it could be obtained with or without facilitation.

2. Vibratory sensation. Vibratory sensation was obtained in a semiquantitative manner proximal to the great toe nailbed and at the medial malleolus. The number of seconds between the striking of the tuning fork and the subject reporting that the vibrations had ceased was recorded.

3. Position sense. The examiner grasped the dominant great toe on the medial and lateral surfaces with the thumb and forefinger. With the subject's eyes closed, a series of 10 small amplitude movements, over a distance of approximately 1 centimeter and a time of about 1 second, were randomly and smoothly administered at irregular intervals. A correct response occurred when the subject perceived the movement and was able identify its direction.

4. Romberg testing. Subjects stood with the feet close together but not touching. The ability to stand without opening the eyes or shifting stance for $30 \mathrm{sec}-$ onds was considered a negative or normal test.

5. Unipedal stance testing. The subjects $(\mathrm{n}=71)$ who underwent this testing (and evaluation with the MDNS, below) were similar to the rest of the subjects but were more-recent participants in balance studies. The subject stood with weight evenly distributed on both feet, which were shoulder width apart, with arms held comfortably at the side. Subjects balanced on the foot of their choice for three separate trials to a maximum of 30 seconds. Failure occurred when the stance foot shifted in any way or the nonstance foot touched the ground.

6. The MDNS. The MDNS is a 0- to 46-point scale (higher score reflecting more severe $\mathrm{PN}$ ) that correlates well with more-extensive neuropathy staging scales. ${ }^{14}$ The scale includes muscle stretch reflexes at 
the biceps, triceps, patella, and Achilles; pinprick sensation at the great toe; ability to perceive the touch of a 10-g monofilament; ability to perceive a $128-\mathrm{Hz}$ tuning fork at the great toe; strength of hand dorsal interossei; and great toe extension and ankle dorsiflexion as determined by manual muscle testing. ${ }^{15}$

\section{Statistical Analysis}

SPSS software was used for all analyses (SPSS Inc., Chicago, IL). Group comparisons included the following. Subjects with one or more falls were compared with those with no falls; subjects with multiple falls were compared with those with none or one fall; and subjects with injuries from falls were compared with subjects without injuries. Descriptive statistics were generated for each of the groups. Group differences in continuous and dichotomous variables were determined with $t$ tests and chi-square analyses, respectively. Logistic regression was performed using the presence of multiple falls or injurious falls as outcomes and demographic, historical, physical examination, and electrodiagnostic variables as predictors.

\section{RESULTS}

Eighty-two subjects were included in the analysis; 52 $(63.4 \%)$ were men. The mean \pm standard deviation height, weight, and body mass index (BMI) were $68.9 \pm$ 4.1 inches, $207.7 \pm 48.3$ pounds, and $30.8 \pm 6.9$, respectively. The subjects had $1.3 \pm 1.0$ comorbidities and took $1.7 \pm 1.3$ medications associated with falls. Forty subjects $(48.8 \%)$ reported a history of at least one fall, $28(34.1 \%)$ reported a history of more than one fall, and $18(22.0 \%)$ reported a history of an injurious fall in the previous 2 years. There was overlap between the multiple and injurious falls groups, with 13 of the 28 subjects with multiple falls reporting injuries and 15 of the 18 subjects who were injured also reporting multiple falls. Trips and stumbles, often under low light conditions, were the most frequent type of fall $(71 \%)$. Turning, leaning over to pick up an object, and falls on steps were other causes of falls.

Factors associated with single and multiple falls were similar, so only results for multiple and injurious falls are reported. Subjects reporting multiple falls had significantly greater BMIs and MDNS scores than subjects reporting none or one fall (Table 1). In addition, subjects with multiple falls demonstrated a trend toward a lower peroneal motor amplitude than subjects with none or one fall. Subjects reporting injurious falls also demonstrated higher BMI and MDNS scores than those not reporting injurious falls. Subjects with injuries from falls also demonstrated decreased ankle vibratory sense and reported increased consumption of medications associated with falls (Table 1). Age was not associated with multiple or injurious falls.

Subjects with abnormal, compared with normal, dorsiflexion strength were more likely to report multiple and injurious falls $(56.0 \%$ vs $23.4 \%, P=.006$, and $44.0 \%$ vs $15.2 \%, P=.008$, respectively). However, multiple or injurious falls did not vary with sex $(28.3 \%$ men vs $43.3 \%$ women, $P=.183$, and $21.2 \%$ men vs $24.1 \%$ women, $P=$ .757 , respectively) or the presence of diabetes mellitus (32\% with vs $37.5 \%$ without diabetes mellitus, $P=.608$, and $26.5 \%$ with vs $15.6 \%$ without diabetes mellitus, $P=$
.248 , respectively). Subjects with abnormal Romberg tests were not more likely than subjects with normal Romberg tests to report multiple or injurious falls $(50.0$ vs $31.3 \%, P=$ .182 , and 14.3 vs $24.2 \%, P=.418$, respectively).

When women were analyzed separately (Table 2), increased BMI remained associated with multiple and injurious falls, but increased MDNS or abnormal dorsiflexion strength were not associated with either fall outcome. Although men reporting falls demonstrated increased BMI, the difference was not significant. However, increased MDNS and abnormal dorsiflexion strength were associated with both fall outcomes (Table 3 ). In addition, men, but not women, who reported multiple and injurious falls demonstrated a decreased UPS than men who had not fallen.

Logistic regression analysis revealed that BMI and MDNS were significant predictors of both fall categories while controlling for age, sex, and number of comorbidities and medications associated with falls (Tables 4 and 5). There was a trend toward medications associated with falls being a predictor of both fall categories. The models predicted $46 \%$ and $48 \%$ of the variability in fall category for multiple and injurious falls, respectively. The addition of mean UPS time did not significantly improve $R^{2}$ values, and $R^{2}$ values were significantly decreased if dorsiflexion strength was substituted for the MDNS (to 29\% and 36\% for multiple and injurious falls, respectively).

\section{DISCUSSION}

The major results of this study suggest two novel conclusions regarding fall risk in older persons with $\mathrm{PN}$; fall risk is increased in those with more severe $\mathrm{PN}$, and fall risk is increased in those with a higher BMI. These two factors were significantly associated with multiple and injurious falls after controlling for age, sex, medications, and comorbidities associated with falls. Nevertheless, when the sexes were analyzed separately, the positive association between BMI and fall risk was seen in women but not men, whereas the positive association between neuropathy severity and fall risk was identified in men but not women. There were trends toward medications associated with falls predicting both fall outcomes, but neither age nor number of comorbidities was associated with either fall outcome in the age group analyzed.

Although the author is unaware of other work that has found that severity of $\mathrm{PN}$ is associated with fall risk, the finding is intuitively appealing and is supported by studies that have identified impairments in lower extremity function that are proportionate to PN severity. For example, Simoneau et al. found that severity of sensory impairment in subjects with diabetic PN was strongly associated with degree of postural instability as detected on a force platform, ${ }^{4}$ and significant correlations between parameters of posturography and peripheral nerve conduction studies have been identified in subjects with diabetic PN. ${ }^{5}$ In men, but not women, distal lower extremity vibrotactile thresholds were found to be the most important variable associated with balance in a large cohort of older persons who presumably represented a spectrum of peripheral nerve health. ${ }^{16}$ Correlations have been identified between PN severity, peak velocity, and isokinetic strength at the ankle and knee. ${ }^{17,18}$ The correlations between PN severity and 
Table 1. Characteristics of Subjects Reporting Multiple Falls Versus No or One Fall and Injurious Falls Versus No History of Injurious Falls

\begin{tabular}{|c|c|c|c|c|c|c|}
\hline \multirow{3}{*}{ Clinical Characteristic } & \multicolumn{3}{|c|}{ History of Multiple Falls } & \multicolumn{3}{|c|}{ History of Injurious Falls } \\
\hline & $\begin{array}{c}\text { Yes } \\
n=28\end{array}$ & $\begin{array}{c}\text { No } \\
n=54\end{array}$ & \multirow[b]{2}{*}{$P$-value* } & $\begin{array}{c}\text { Yes } \\
n=18\end{array}$ & $\begin{array}{c}\text { No } \\
n=64\end{array}$ & \multirow[b]{2}{*}{$P$-value ${ }^{*}$} \\
\hline & \multicolumn{2}{|c|}{ Mean \pm Standard Deviation } & & \multicolumn{2}{|c|}{ Mean \pm Standard Deviation } & \\
\hline Age & $64.5 \pm 9.5$ & $66.3 \pm 9.0$ & .396 & $63.8 \pm 10.2$ & $66.3 \pm 8.9$ & .326 \\
\hline Body mass index & $33.6 \pm 8.9$ & $29.3 \pm 5.0$ & .023 & $34.1 \pm 8.3$ & $29.8 \pm 6.2$ & .018 \\
\hline Sural amplitude $(\mu \mathrm{V})$ & $0.7 \pm 1.6$ & $1.0 \pm 1.5$ & .444 & $0.4 \pm 1.1$ & $1.1 \pm 1.6$ & .064 \\
\hline Peroneal amplitude (mV) & $0.9 \pm 1.4$ & $1.7 \pm 1.6$ & .050 & $1.4 \pm 1.9$ & $1.4 \pm 1.5$ & .990 \\
\hline Vibration toe (seconds) & $2.6 \pm 3.3$ & $2.8 \pm 3.8$ & .850 & $2.0 \pm 2.3$ & $2.9 \pm 3.9$ & .377 \\
\hline Vibration ankle (seconds) & $5.9 \pm 4.5$ & $7.9 \pm 5.7$ & .109 & $4.9 \pm 4.1$ & $7.9 \pm 5.5$ & .034 \\
\hline Medications & $1.9 \pm 1.5$ & $1.5 \pm 1.2$ & .325 & $2.3 \pm 1.7$ & $1.5 \pm 1.1$ & .026 \\
\hline Comorbidities & $1.2 \pm 0.9$ & $1.3 \pm 1.1$ & .622 & $1.6 \pm 1.3$ & $1.2 \pm 0.9$ & .179 \\
\hline
\end{tabular}

${ }^{*} t$ test.

impairments noted in these studies suggest that patients with more severe PN would be at greater fall risk, one of the major findings of the present study. It is notable that falls were associated with increased severity of PN as measured by the MDNS, as opposed to other isolated measures of PN severity such as vibratory sensation, position sense, or nerve conduction amplitudes. This is likely because the MDNS, unlike the other measures mentioned, allows an evaluation of afferent and efferent peripheral nerve function, suggesting that both are important to postural stability. The importance of both sensory and motor peripheral nerve function is also supported by the fact that abnormal ankle dorsiflexion strength was associated with falls in univariate analysis but not with logistic regression when MDNS was included, and, when ankle dorsiflexion strength was substituted for MDNS, the models yielded substantially decreased pseudo $R^{2}$ values.

The positive association between fall risk and increased BMI may be explained biomechanically. Success- ful standing and ambulation are based on the patient controlling the center of mass and maintaining it over (during standing) or just anterior and medial to (during walking) a functional base of support. ${ }^{19}$ This requires precise feedback from the visual, vestibular, and somatosensory afferent systems. However, previous work has demonstrated that subjects with PN have impairments in proprioceptive thresholds of ankle inversion/eversion ${ }^{20}$ and plantar/dorsiflexion. ${ }^{21}$ As a result, a patient with PN would be expected to make errors that allow the center of mass to migrate outside the functional base of support. Obese persons may be even more inclined to make errors, particularly in the frontal plane, because of thigh and hip mass. ${ }^{22}$ Furthermore, for a given magnitude of error, the obese patient's center of mass would have increased momentum, which would be more difficult to arrest and control than the momentum generated by a similar error in a thin person with $\mathrm{PN}$, particularly given the known impairments in ankle strength and rate of strength development in subjects with

Table 2. Characteristics of Women $(n=30)$ by Fall Outcome

\begin{tabular}{|c|c|c|c|c|c|c|}
\hline \multirow[b]{2}{*}{ Parameter } & \multicolumn{3}{|c|}{ Multiple Falls } & \multicolumn{3}{|c|}{ Injurious Falls } \\
\hline & $\begin{array}{c}\text { Yes } \\
n=13\end{array}$ & $\begin{array}{c}\text { No } \\
\mathrm{n}=17\end{array}$ & $P$-value ${ }^{*}$ & $\begin{array}{c}\text { Yes } \\
\mathrm{n}=7\end{array}$ & $\begin{array}{c}\text { No } \\
n=23\end{array}$ & $P$-value* \\
\hline Age, mean $\pm S D$ & $62.5 \pm 7.4$ & $66.3 \pm 9.0$ & .223 & $59.9 \pm 6.7$ & $66.3 \pm 8.6$ & .081 \\
\hline Body mass index, mean $\pm S D$ & $37.4 \pm 8.9$ & $30.7 \pm 5.6$ & .018 & $39.8 \pm 6.5$ & $31.7 \pm 7.4$ & .015 \\
\hline Mean unipedal stance (seconds), mean $\pm S D$ & $4.6 \pm 5.5$ & $4.5 \pm 3.0$ & .606 & $4.7 \pm 6.3$ & $4.0 \pm 2.9$ & .730 \\
\hline Michigan Diabetes Neuropathy score, mean \pm SD & $14.8 \pm 6.7$ & $15.1 \pm 4.9$ & .882 & $16.5 \pm 5.8$ & $14.6 \pm 5.6$ & .471 \\
\hline Dorsiflexion strength (\% abnormal) & 46.2 & 37.5 & .638 & 62.5 & 35.0 & 184 \\
\hline
\end{tabular}

${ }^{\star} t$ test.

$\mathrm{SD}=$ standard deviation. 
Table 3. Characteristics of Men $(n=52)$ by Fall Outcome

\begin{tabular}{|c|c|c|c|c|c|c|}
\hline \multirow[b]{2}{*}{ Parameter } & \multicolumn{3}{|c|}{ Multiple Falls } & \multicolumn{3}{|c|}{ Injurious Falls } \\
\hline & $\begin{array}{c}\text { Yes } \\
n=15\end{array}$ & $\begin{array}{c}\text { No } \\
\mathrm{n}=37\end{array}$ & $P$-value* & $\begin{array}{c}\text { Yes } \\
n=11\end{array}$ & $\begin{array}{c}\text { No } \\
n=41\end{array}$ & $P$-value* \\
\hline Age, mean $\pm S D$ & $66.2 \pm 10.9$ & $66.3 \pm 9.1$ & .981 & $66.4 \pm 11.4$ & $66.2 \pm 9.1$ & .965 \\
\hline Body mass index, mean $\pm S D$ & $30.3 \pm 7.7$ & $28.6 \pm 4.7$ & .343 & $30.5 \pm 7.4$ & $28.7 \pm 5.2$ & .364 \\
\hline Mean unipedal stance (seconds), mean \pm SD & $3.7 \pm 3.3$ & $7.8 \pm 8.6$ & .025 & $3.5 \pm 3.3$ & $7.7 \pm 8.3$ & .025 \\
\hline Michigan Diabetes Neuropathy score, mean \pm SD & $25.7 \pm 7.5$ & $16.1 \pm 4.2$ & $<.001$ & $24.6 \pm 7.9$ & $16.1 \pm 4.3$ & $<.001$ \\
\hline Dorsiflexion strength (\% abnormal) & 66.7 & 16.1 & .001 & 60.0 & 21.2 & .019 \\
\hline
\end{tabular}

${ }^{*} t$ test.

$\mathrm{SD}=$ standard deviation.

PN. ${ }^{18,23}$ Other work that has found obesity-associated impairments in standing ${ }^{24}$ and dynamic balance support this rationale. ${ }^{25}$ The stronger association between increased BMI and falls in women may relate to sex-based differences in typical fat distribution patterns, with men gathering fat in a less-destabilizing mid-line location and women in relatively more-destabilizing and lateral hip and thigh locations.

The association between obesity and injurious falls in this study was contrary to the findings of others that identified an association between decreased BMI and risk for fall injury, ${ }^{26-28}$ particularly fracture, which has been attributed to decreased bone density and decreased mechanical protection of bones in older persons with a lower BMI. However, the data reported here suggest that, at least in women with $\mathrm{PN}$, the biomechanical disadvantages that predispose obese patients with PN to fall overwhelm whatever advantage such patients may have in terms of decreased risk for injury upon impact. This disadvantage may extend to young women as well, given that a large community study demonstrated an increased risk of injurious falls in women younger than 45 with a BMI of 30 or greater. ${ }^{29}$

Most other work that has investigated PN and postural stability has involved diabetic PN exclusively. In this study, $32(39.0 \%)$ of the subjects did not have diabetes mellitus. In this group, any fall, multiple falls, and injurious falls were reported with a frequency that was not dif- ferent in the subjects with diabetes mellitus. In addition, other work has demonstrated that mobility impairments and decreased isokinetic muscle strength in the lower extremities of patients with diabetes mellitus is associated with PN but not simply with the presence of diabetes mellitus or other complications such as nephropathy or retinopathy. ${ }^{17,30}$ These findings suggest that $\mathrm{PN}$, rather than the simple presence of diabetes mellitus, leads to impairment in balance and increased fall risk in older persons.

It would be clinically convenient if one of the tests of clinical balance could reliably detect patients with PN who are at risk for falls. Unfortunately, such was not the case. The data suggest that mean UPS time is decreased in men reporting falls, but the variability of the data prevented the identification of a clear cutoff value that might be helpful to clinicians, and the relationship was not present in the women, who bear the greatest burden from falls. However, UPS time may deserve further study as an indicator of fall risk in patients with $\mathrm{PN}$, at least with regard to men, given that other work has found a decreased UPS time to be associated with falls in men in a large cohort of community-dwelling older persons. ${ }^{31}$ Romberg testing was not associated with any of the fall outcomes and does not appear useful in detecting patients with $\mathrm{PN}$ who are at increased risk for falls. A clinical test focused more on medial-lateral stability, such as tandem or near-tandem balance, may be more effective than the tests used in this study. ${ }^{32}$ In addition, the balance tasks used were relatively

Table 4. Results of Logistic Regression for the Presence of Multiple Falls

\begin{tabular}{lccc}
\hline \multicolumn{1}{c}{ Predictor Variable } & Coefficient & $P$-value & OR (95\% Cl) \\
\hline Age & -0.026 & .54 & $0.97(0.90-1.06)$ \\
Sex & -0.767 & .354 & $0.46(0.092-2.35)$ \\
Body mass index & 0.137 & .026 & $1.15(1.02-1.29)$ \\
Michigan Diabetes Neuropathy score & 0.174 & .0058 & $1.19(1.05-1.35)$ \\
Comorbidities & 0.052 & .89 & $0.95(0.46-1.95)$ \\
Medications & 0.545 & .098 & $1.72(0.90-3.29)$ \\
\hline
\end{tabular}

Note: $R^{2}=0.458$.

$\mathrm{CI}=$ confidence interval; $\mathrm{OR}=$ odds ratio. 
Table 5. Results of Logistic Regression for the Presence of Injurious Falls

\begin{tabular}{lcrr}
\hline \multicolumn{1}{c}{ Predictor Variable } & Coefficient & P-value & OR (95\% Cl) \\
\hline Age & 0.001 & .99 & $1.01(0.92-1.09)$ \\
Sex & -0.171 & .848 & $0.843(0.15-4.80)$ \\
Body mass index & 0.12 & .041 & $1.13(1.01-1.27)$ \\
Michigan Diabetes Neuropathy score & 0.22 & .0042 & $1.24(1.07-1.44)$ \\
Comorbidities & 0.38 & .32 & $1.46(0.69-3.08)$ \\
Medications & 0.58 & .076 & $1.79(0.94-3.41)$ \\
\hline
\end{tabular}

$\mathrm{CI}=$ confidence interval; $\mathrm{OR}=$ odds ratio.

static and likely do not reflect patient performance under more-dynamic conditions of locomotion, an important limitation given the specificity of balance skills ${ }^{33}$ and the fact that most of the patients in this study fell during ambulation.

The strength of the conclusions of this study must be tempered by the study's limitations. The subjects were all aware that they had some neurological symptom involving the lower extremities, although not necessarily PN, because their physicians referred most of them to the electrodiagnostic laboratory for evaluation. This could bias the population studied. They may have had more-severe or more-symptomatic disease or may have been more health conscious and willing to see a physician; therefore, it is not possible to extend the findings to persons with asymptomatic PN. Recall bias is likely, and the retrospective recording of falls may have underestimated the actual prevalence. However, this seems less likely for falls that cause injury, given the emotional effect of such events, and it is therefore reassuring that the findings in this study are similar with regard to all three outcomes: at least one fall, multiple falls, and injurious falls. Furthermore, the overall incidence of falls in this known high-risk group of community dwellers was about $50 \%$, which is, appropriately, greater than the incidence reported in other studies of older persons in the community not known to be at increased risk for falls. ${ }^{34,35}$

The results have clinical ramifications. In patients with PN, those who are obese and have more-severe disease appear at the greatest risk for falls and injurious falls. The severity of PN seems to apply particularly to men, whereas the degree of obesity appears to affect women more strongly. Age was not associated with falls in this group aged 50 to 85 when other factors were taken into account. Therefore, application of techniques to reduce fall risk ${ }^{36,37}$ should be applied to patients with $\mathrm{PN}$ - even if they are relatively young-with special attention to obese women and men with more-severe PN. Although the data do not allow comment on the effect of weight loss on postural stability in patients with $\mathrm{PN}$, a recommendation to lose weight would be clinically prudent given the other associated benefits.

\section{REFERENCES}

1. Judd MA, Roberts CA. Fracture patterns at the Medieval Leper Hospital in Chichester. Am J Phys Anthropol 1998;105:43-55.

2. Ashton-Miller JA, Yeh MW, Richardson JK et al. A cane reduces loss of balance in patients with peripheral neuropathy: Results from a challenging unipedal balance test. Arch Phys Med Rehabil 1996;77:446-452.

3. Richardson JK, Ashton-Miller JA, Lee SG et al. Moderate peripheral neurop- athy impairs weight transfer and unipedal balance in the elderly. Arch Phys Med Rehabil 1996;77:1152-1156.

4. Simoneau GG, Ulbrecht JS, Derr JA et al. Postural instability in patients with diabetic sensory neuropathy. Diabetes Care 1994;17:1411-1421.

5. Uccioli L, Giacomini PG, Monticone G et al. Body sway in diabetic neuropathy. Diabetes Care 1995;18:339-344.

6. Richardson JK, Hurvitz EA. Peripheral neuropathy. A true risk factor for falls. J Gerontol A Biol Sci Med Sci 1995;50A:M211-M215.

7. Cavanagh PR, Derr JA, Ulbrecht JS et al. Problems with gait and posture in neuropathic patients with insulin-dependent diabetes mellitus. Diabetes Med 1992;9:469-474.

8. Richardson JK, Ching C, Hurvitz EA. The relationship between electromyographically documented peripheral neuropathy and falls. J Am Geriatr Soc 1992;40:1008-1012.

9. Harris MI, Flegal KM, Cowie CC et al. Prevalence of diabetes, impaired fasting glucose, and impaired glucose tolerance in U.S. Adults: The Third National Health and Nutrition Examination Survey, 1988-1994. Diabetes Care 1998;21:518-524.

10. Franklin GM, Kahn LB, Baxter J et al. Sensory neuropathy in non-insulindependent diabetes mellitus. The San Luis Valley Diabetes Study. Am J Epidemiol 1990;131:633-643.

11. King MB, Tinetti ME. A multifactorial approach to reducing injurious falls. Clin Geriatr Med 1996;12:745-759.

12. Monane M, Avorn J. Medications and falls: Causation, correlation and prevention. Clin Geriatr Med 1996;12:847-858.

13. O'Keefe ST, Smith T, Valacio R et al. A comparison of two techniques for ankle jerk assessment in elderly subjects. Lancet 1994;344:1619-1620.

14. Feldman EL, Stevens MJ, Thomas PK et al. A practical two-step quantitative clinical and electrophysiological assessment for the diagnosis and staging of diabetic neuropathy. Diabetes Care 1994;17:1281-1289.

15. Daniels L, Worthingham C. Muscle Testing. Techniques of Manual Examination, 5th Ed. Philadelphia, PA: W.B. Saunders, 1986.

16. Era P, Schroll M, Ytting $\mathrm{H}$ et al. Postural balance and its sensory-motor correlates in 75-year-old men and women: A cross-national comparative study. J Gerontol A Biol Sci Med Sci 1996;51A:M53-M63.

17. Andersen H, Mogensen PH. Disordered mobility of large joints in association with neuropathy in patients with long-standing insulin-dependent diabetes. Diabetes Med 1997; 14:221-227.

18. Andersen H, Poulsen PL, Mogensen CE et al. Isokinetic muscle strength in long-term IDDM patients in relation to diabetic complications. Diabetes 1996;45:440-445.

19. Winter DA, ed. The Biomechanics and Motor Control of Human Gait: Normal, Elderly and Pathological, 2nd Ed. Waterloo, Canada: Waterloo Press, 1991, pp 75-85.

20. Van den Bosch CG, Gilsing MG, Lee SG et al. Peripheral neuropathy effect on ankle inversion and eversion detection thresholds. Arch Phys Med Rehabil $1995 ; 76: 850-856$.

21. Simoneau GG, Derr JA, Ulbrecht JS et al. Diabetic sensory neuropathy effect on ankle joint movement perception. Arch Phys Med Rehabil 1996;77:453-460.

22. McGraw B, McClenaghan BA, Williams HG et al. Gait and postural stability in obese and nonobese prepubertal boys. Arch Phys Med Rehabil 2000;81: 484-489.

23. Gutierrez EM, Helber MD, Dealva D et al. Mild diabetic neuropathy affects ankle motor function. Clin Biomech 2001;16:522-528.

24. Lichtenstein MJ, Shields SL, Shiavi RG et al. Clinical determinants of biomechanics platform measures of balance in aged women. J Am Geriatr Soc 1998;36:996-1002.

25. Jadelis K, Miller ME, Ettinger WH Jr. et al. Strength, balance, and the modifying effects of obesity and knee pain: Results from the Observational Arthritis Study in Seniors (OASIS). J Am Geriatr Soc 2001;49:884-891. 
26. Farmer ME, Harris T, Madans JH et al. Anthropometric indicators and hip fracture: The NHANES I epidemiologic follow-up study. J Am Geriatr Soc 1989;37:9-16.

27. Meyer HE, Tverdal A, Falch JA. Risk factors for hip fracture in middle-aged Norwegian women and men. Am J Epidemiol 1993;137:1203-1211.

28. Wickham CA, Walsh K, Cooper C et al. Dietary calcium, physical activity, and risk of hip fracture: A prospective study. BMJ 1989;299:889-892.

29. Malmivaara A, Heliovaara M, Knekt P et al. Risk factors for injurious falls leading to hospitalization or death in a cohort of 19,500 adults. Am J Epidemiol 1993;138:384-394.

30. Resnick HE, Stansberry KB, Harris TB et al. Diabetes, peripheral neuropathy and old age disability. Muscle Nerve 2002;25:43-50.

31. Vellas BJ, Wayne SJ, Garry PJ et al. A two-year longitudinal study of falls in 482 community-dwelling elderly adults. J Gerontol A Biol Sci Med Sci 1998; 53A:264-274.
32. Lord SR, Rogers MW, Howland A et al. Lateral stability, sensorimotor function and falls in older people. J Am Geriatr Soc 1999;47:1077-1081.

33. Horak FB, Henry SM, Shumway-Cook A. Postural perturbations. New insights for treatment of balance disorders. Phys Ther 1997;77:517-533.

34. Lord SR, Ward JA, Williams P et al. Physiological factors associated with falls in older community-dwelling women. J Am Geriatr Soc 1994;42:11101117.

35. Tinetti ME, Speechley M, Ginter SF. Risk factors for falls among elderly persons living in the community. N Engl J Med 1988;319:1701-1707.

36. Close J, Ellis M, Hooper R et al. Prevention of falls in the elderly trial (PROFET): A randomized controlled trial. Lancet 1999;353:93-97.

37. Province MA, Hadley EC, Hornbrook MC et al. The effects of exercise on falls in elderly patients: A preplanned meta-analysis of the FICSIT Trials. Frailty Injuries: Cooperative Studies Intervention Techniques. JAMA 1995; 273:1341-1347. 